Ann. Génét. Sél. anim., I978, 10 (2), I6I-I70.

\title{
Le Caryotype du cheval domestique (Equus caballus) de l'âne (Equus asinus) et du mulet par la méthode des bandes $\mathrm{G}$
}

\author{
E. P. CRIBIU et Annamaria DE GIOVANNI* \\ avec la collaboration technique de Jeannine BoscuER \\ Labovatoive de Cytogénétique U.N.C.E.I.A., I.N.R.A. \\ Centre National de Recherches Zootechniques \\ 78350 Jouy-en-Josas, France \\ * Istituto di Zootecnia Generale \\ Facoltà di Agraria, Via Celoria 2 \\ Milano, Italie
}

\begin{abstract}
Résumé
L'hétérochromatine constitutive est située dans les régions centromériques de tous les chromosomes du caryotype du cheval domestique (Equus caballus) à l'exception de la $\mathrm{II}^{\mathrm{e}}$ paire qui n'en possède pas. Le chromosome $X$ présente en plus d'une bande péricentromérique une bande interstitielle d'hétérochromatine constitutive sur la partie médiane du grand bras. Le gonosome $\mathrm{Y}$ est presque entièrement hétérochromatique. Chez l'âne (Equus asinus), l'hétérochromatine constitutive est tantôt en position péricentromérique, tantôt terminale, tantôt interstitielle et même complètement absente. Le chromosome $\mathrm{X}$ possède une bande interstitielle d'hétérochromatine constitutive sur la partie médiane des bras longs. Chez le mulet, les deux compléments paternels et maternels sont facilement identifiés.
\end{abstract}

\section{Introduction}

Le nombre chromosomique exact du cheval domestique (Equus caballus) fut établi pour la première fois par ROTHEFELS et al. en I 959 puis confirmé, par la suite, par Trujillo et al. (r962), SASAKI et Makino (r962), Makino et al (I963). Celui de l'Ane (Equus asinus) et du Mulet furent étudiés par BENIRSCHKE et al. (I962), Trujilio et al. (I962), Kofman-Alfaro et al. (I966) et Fildridge et BLAZAK (I976).

La technique de marquage $\mathrm{C}$ appliquée sur les chromosomes du cheval par MELchior et HoHn (I976) et BUCKLAND et al. (I976) et sur les chromosomes de l'âne par RYDER et al. (I978) colore spécifiquement un type particulier de chro- 
matine : l'hétérochromatine constitutive qui serait composée d'après Comings et MatTocia (I972) d'un ADN hautement répétitif.

Le but de ce travail est l'étude du caryotype du Cheval, de 1'Ane et du Mulet à l'aide du marquage $C$.

\section{Matériel et méthodes}

Nous avons disposé d'une dizaine de poneys et de chevaux de selle français, de 5 ânes du Poitou ( 4 mâles et I femelle) et de 3 mulets ( 2 mâles et I femelle). Sur chaque animal ont été pratiqués deux prélèvements, l'un sur tube sec afin de pouvoir disposer du sérum et l'autre sur tube hépariné.

Les cultures de leucocytes ont été effectuées avec du sérum autologue selon la méthode de Moorhead et al. (I960). Après un blocage de I h 30 à la colchicine, un choc hypotonique de 20 minutes et trois fixations d'une heure, la suspension cellulaire a été étalée sur des lames préalablement refroidies. Celles-ci ont ensuite été séchées à l'air, sans flamme, puis stockées pendant plusieurs jours. Quelques lames ont ensuite été colorées au Giemsa tandis que les autres ont été traitées selon la méthode de Sumner (I972) légèrement modifiée : les lames, après un rinçage rapide dans l'eau déminéralisée, sont plongées directement dans une solution à $5 \mathrm{p}$. I Ioo d'hydroxyde de baryum chauffée à $40^{\circ} \mathrm{C}$ pendant $\mathrm{I} 5$ minutes. Elles sont ensuite abondemment rincées à l'eau du robinet, puis à 1'eau déminéralisée pour être mises à incuber pendant I h $45 \mathrm{mn}$ à $5^{\circ}{ }^{\circ} \mathrm{C}$ dans une solution $2 \mathrm{XSSC}(0,3 \mathrm{M}$ de chlorure de sodium $0,03 \mathrm{M}$ de citrate trisodique) à $\mathrm{pH} 7$. Après un rinçage rapide dans l'eau déminéralisée, les lames sont colorées dans une solution de Giemsa à 4 p. Ioo pendant 7 minutes.

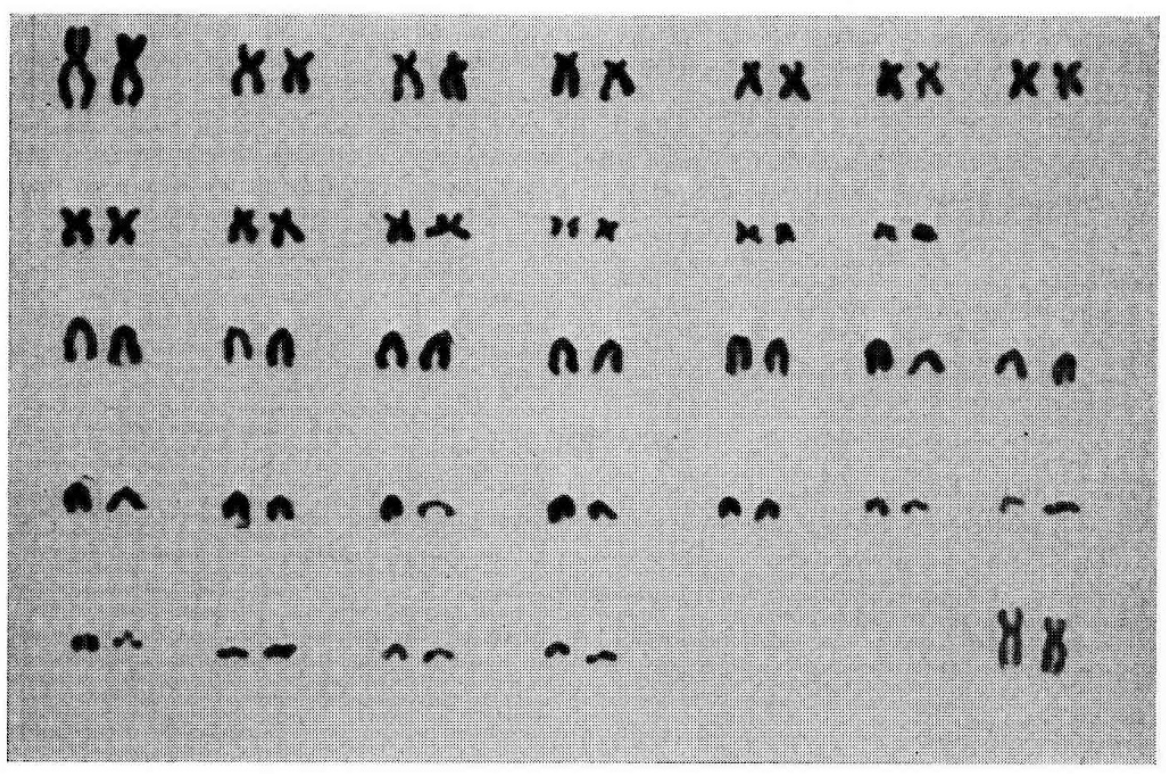

FIG. I. - Caryotype temelle du cheval domestique. Karyotype of the mave. 
Les observations ont été effectuées à l'aide d'un microscope Leitz-Ortholux et les photographies prises avec une caméra Leitz Orthomat sur un film Kodak Microfile.

\section{Résultats}

\section{Equus caballus}

Le nombre diploïde de Equus caballus est $2 n=64$. Son caryotype est composé de I3 paires d'autosomes méta-submétacentriques, I 8 paires d'autosomes acrocentriques, un chromosome sexuel $\mathrm{X}$ submétacentrique dont la taille est intermédiaire entre la première et la deuxième paire des plus grands méta-submétacentriques et un gonosome $\mathrm{Y}$ acrocentrique de petite taille (fig. I).

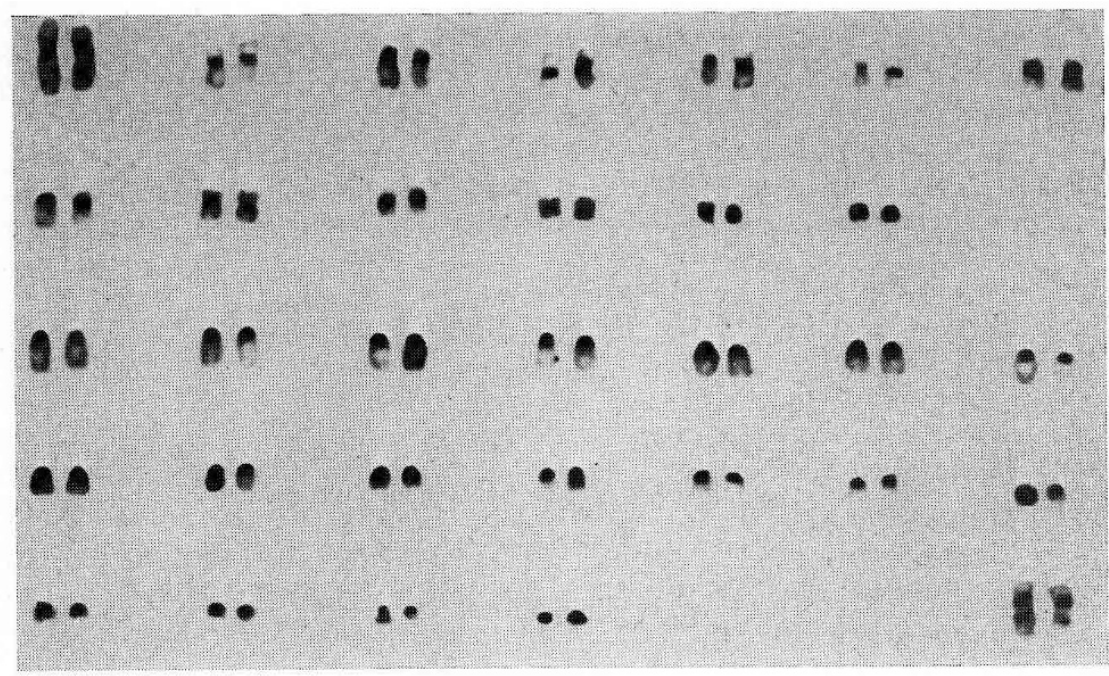

FIG. 2. - Caryotype à bande $C$ du cheval domestique.

$C$-banding karyotype of the mare.
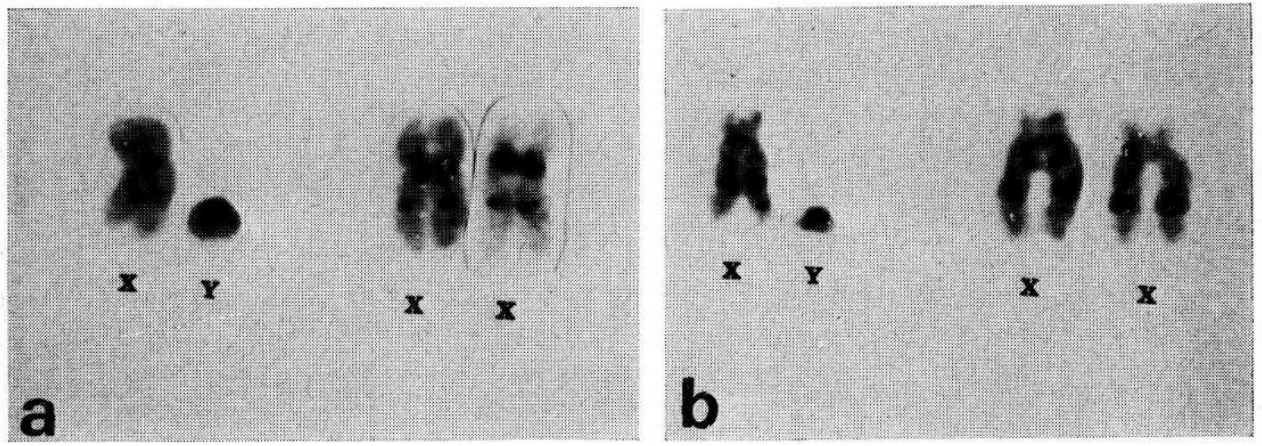

FIG. 3. - Marquage $C$ des chromosomes sexuels du cheval (a) et de l'âne (b). $C$-banding pattern of the sex chromosomes of the Horse (a) and the Donkey (b). 


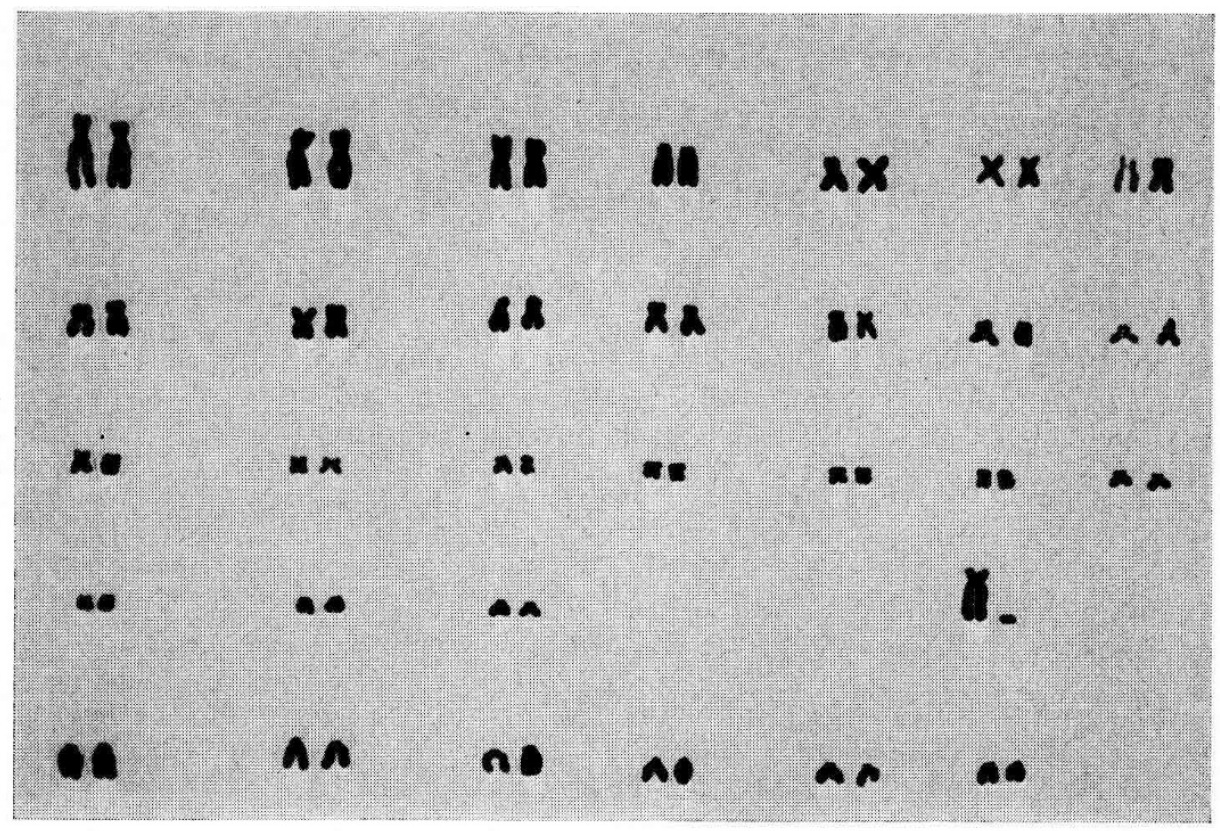

FIG. 4. - Caryotype mâle de l'Ane.

Karyotype of the male Donkey.

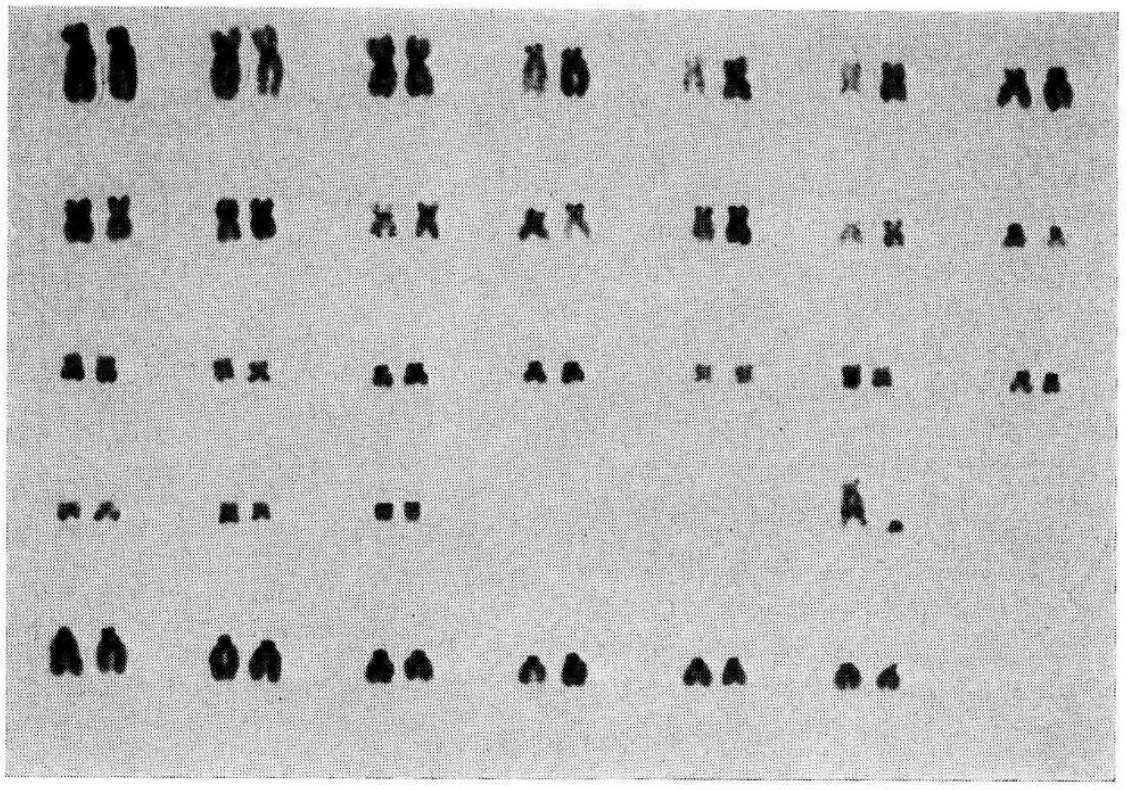

Frg. 5. - Caryotype ì bande $C$ de l'âne.

$C$-banding karyotype of male Donkey. 


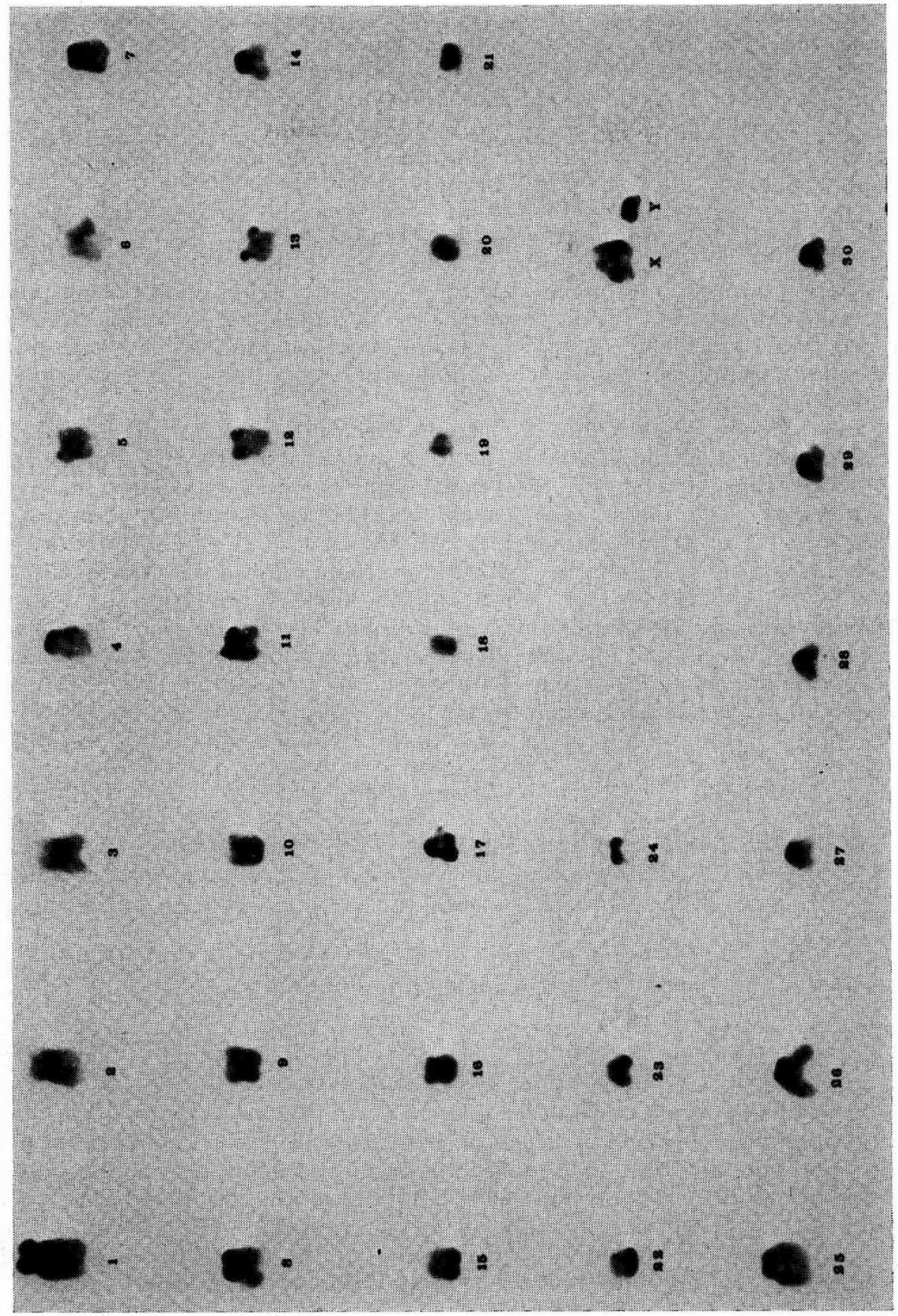

FIG. 6. - Caryotype à bande $C$ de l'âne (Un chromosome pour chaque paire du caryotype a été représenté et les numéros affectés à chacun ont été donnés arbitrairement pour permettre une meilleure compréhension du texte).

$C$-banding karyotype of the Donkey (One chromosome of each pair is put. The numbers arbitrarly assigned are given to let the understanding of the text). 
L'hétérochromatine constitutive, présente une disposition semblable sur tous les autosomes, au niveau de la région centromérique, à l'exception de la paire $\mathrm{n}^{\mathrm{o}}$ I I qui semble ne pas en posséder (fig. 2). Le gonosome $\mathrm{X}$ montre une région plus intensément colorée au niveau du centromère et sur la partie médiane du bras long. Le chromosome $\mathrm{Y}$ possède de l'hétérochromatine constitutive sur pratiquement toute sa longueur à l'exception de la région télomérique (fig. 3).

\section{Equus asinus}

L'âre possède $2 n=62$ chromosomes dont 24 paires d'autosomes métasubmétacentriques, 6 paires d'autosomes acrocentriques, un gonosome $\mathrm{X}$ submétacentrique de taille comprise entre la troisième et la quatrième paire des grands méta-submétacentriques et un chromosome sexuel $\mathrm{Y}$ très petit et submétacentrique (fig. 4).

La technique du marquage $\mathrm{C}$ met en évidence une très grande diversité en ce qui concerne l'emplacement de l'hétérochromatine constitutive sur les autosomes. Les paires I, 7 et I 7 présentent des régions intensément colorées au niveau du centromère et des parties télomériques des tras. Sur les paires 4, 8, II, I2, I3, I $5,22,23$ et 24 on remarque des zones colorées sur la partie terminale des chromatides. De l'hétérochromatine péricentromérique semble se trouver sur les paires $2,3, \mathrm{IO}, \mathrm{r} 4,2 \mathrm{I}, 25,26,27,28,29$ et 30 . Les autres paires restantes paraissent ne pas posséder de marquage $\mathrm{C}$ ou un marquage trop faible pour être détecté (fig. 5 et 6 ). Le chromosome sexuel $X$ possède une bande d'hétérochromatine sur la partie médiane du bras long. Le gonosome $\mathrm{Y}$ présente une zone hétérochromatique au niveau du centromère (fig. 3).

\section{Le Mulet}

Le nombre diploïde du Mulet est de $2 n=63$. Son caryotype représenté sur la fig. 7 met en évidence la présence des deux compléments paternels et maternels.

\section{Discussion}

\section{Equus caballus}

Les I3 paires d'autosomes méta-submétacentriques présentent dans la région péricentromérique de l'hétérochromatine constitutive à l'exception de la II ${ }^{\mathrm{e}}$ paire qui n'en possède pas. Bien que de tels chromosomes soient relativement rares chez les mammifères, il en a été trouvés chez certaines espèces tel que des sous-espèces du rat (RAman et Sherma, I974) et chez l'Opossum (Sinna et al., I972).

$\mathrm{Si}$ la disposition uniforme de l'hétérochromatine constitutive sur tous les autosomes acrocentriques ne peut servir à leur identification, on constate toutefois que le chromosome $\mathrm{Y}$ jusqu'alors très difficilement reconnaissable en raison de sa morphologie semblable aux autosomes acrocentriques est très facilement détec. table à l'aide du marquage $\mathrm{C}$. Cet aspect particulier a été confirmé par le mar. quage G (CHANDLEY et al., I975) qui montre une bande sombre sur sa partie télomérique. 
La présence d'une bande interstitielle d'hétérochromatine constitutive sur la partie médiane $d u$ bras long permet de distinguer le chromosome $\mathrm{X}$ parmi les premières paires de méta-submétacentriques.

\section{Equus asinus}

Si le nombre de 62 chromosomes chez l'âne est admis par tous les auteurs, il n'en est pas de même de leur morphologie, car le nombre d'acrocentriques, de métacentriques ou de submétacentriques varie d'une publication à l'autre. BENIRSCHKE et al. (I962) pensent que le caryotype de l'Ane comporte entre I9

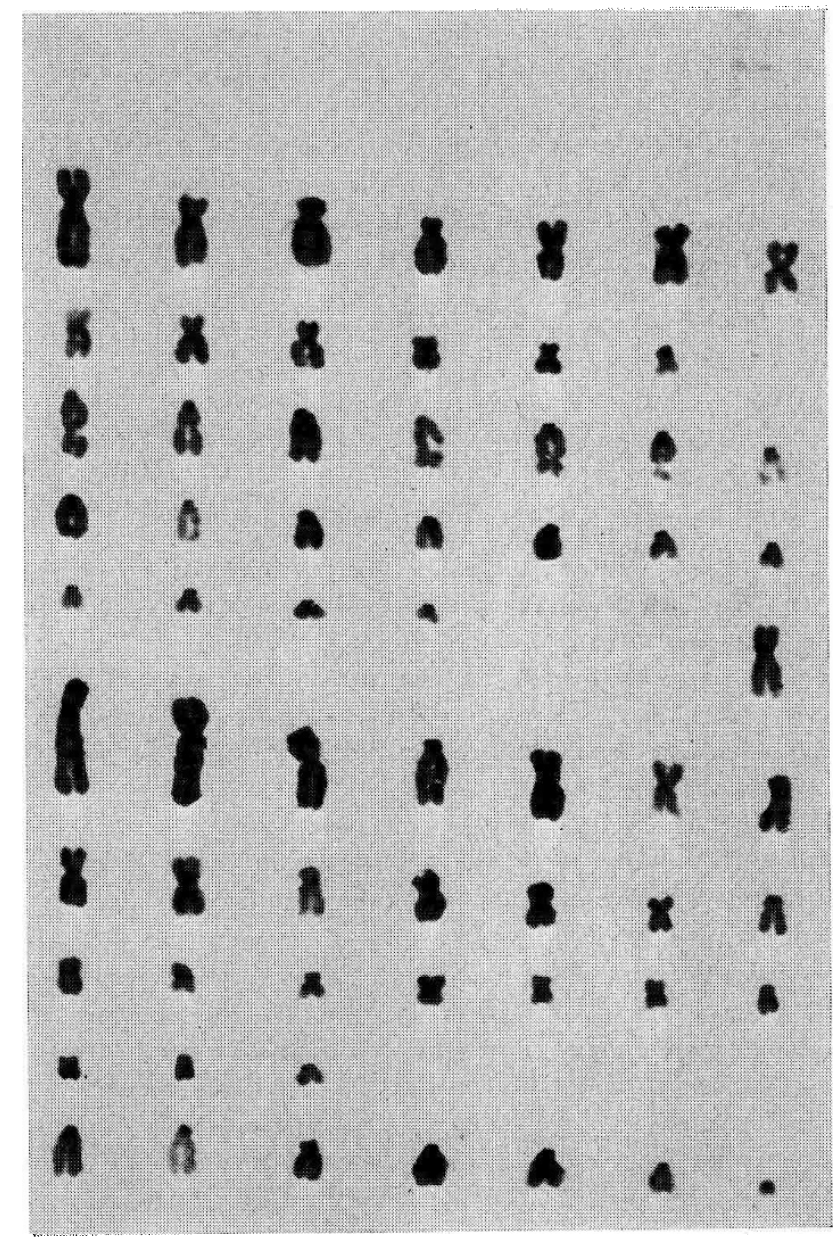

FIG. 7. - Caryotype à bande $C$ du mulet.

C-banding karyotype of the male mule.

et 2 I paires d'autosomes méta-submétacentriques, TrujILLo et al. (I962), KoFMANAlfFaro et $a$. (I966) en dénombre I9 paires tandis que ELDRIDGE et BLAZAK (I976) en trouvent 24 paires. Nos résultats confirment 1'hypothèse de ces derniers. En 
effet, lorsque l'on possède des chromosomes suffisamment despiralisés, l'on peut observer 24 paires d'autosomes méta-submétacentriques. L'étude du caryotype à l'aide du marquage $\mathrm{C}$ confirme ces résultats puisque sur certains petits chromosomes, qui autrefois, étaient considérés comme acrocentriques on voit des bandes $\mathrm{C}$ en position terminale sur les petits bras. Le chromosome $\mathrm{Y}$ apparaît, lui aussi, dans certaines métaphases, submétacentrique.

Les chromosomes de l'Ane présentent une remarquable diversité quant au marquage $\mathrm{C}$. L'hétérochromatine constitutive est, en effet, tantôt en position péricentromérique, tantôt terminale, tantôt interstitielle et même complétement absente. L'origine de l'hétérochromatine constitutive terminale ou interstitielle s'explique d'après Hsu et ARRIGHI (I97I) par deux hypothèses : une translocation d'un acrocentrique sur la partie terminale d'un métacentrique ou une inversion comprenant l'hétérochromatine péricentromérique.

Le gonosome $X$ qui ne possède pas d'hétérochromatine péricentromérique mais une bande interstitielle sur la partie médiane du bras long est aisément identifiable. De par sa morphologie et son extrême petite taille, le chromosome Y est lui aussi facilement reconnaissable.

En prenant comme critère la taille, la morphologie et la distribution de 1'hétérochromatine constitutive, il est possible d'individualiser presque tous les chromosomes de 1'Ane, à 1'exception des paires I8, I9 et 20.

\section{Le Mulet}

Le Mulet, comme 1'on pouvait s'y attendre, comporte 63 chromosomes parmi lesquels l'on remarque un complément haploïde du cheval et un complément haploïde de 1'âne. La technique de marquage $\mathrm{C}$ permet, à l'exception d'un petit nombre de paires chromosomiques, une bonne distinction entre les deux compléments. A la lumière des résultats donnés ici, il semble que les deux espèces Equus asinus et Equus caballus aient surtout évolué au point de vue chromosomique par inversions péricentriques comme semble 1'attester la présence d'hétérochromatine constitutive télomérique. Cependant certaines paires telles que la paire $n^{\circ}$ I et la paire $n^{0} 7$ de l'âne pourraient différer de celle du cheval par d'autres types de remaniements telles que les translocations.

Sur les chromosomes $\mathrm{X}$ des deux espèces, l'on retrouve la bande interstitielle située sur la partie médiane du bras long. Cependant, 1'Ane a perdu 1'hétérochromatine constitutive péricentromérique au cours de son évolution. Les deux gonosomes $\mathrm{Y}$ semblent, eux aussi, avoir évolué différemment du point de vue morphologique et distribution d'hétérochromatine constitutive. Une cause possible de la stérilité de la mule et du mulet pourrait être, selon BenIRschke (I 967) d'ordre chromosomique. En effet, les différences importantes du point de vue morphologique et distribution d'hétérochromatine constitutive entre les compléments chromosomiques du cheval domestique et de l'âne entraîneraient des anomalies d'appariement dans les spermatocytes primaires à la méiose (CHANdLEY et al., I974). 


\section{Remerciements}

Nous remercions le $\mathrm{D}^{\mathrm{r}} \mathrm{C}$. P. PopESce (Laboratoire de Cytogénétique U.N.C.E.I.A.-I.N.R.A , C.N.R.Z., Jouy-en-Josas, France) pour son aide précieuse dans la réalisation de ce travail, ainsi que le $D^{r}$ N. S. Fechinfrmer (Animal Reproduction Research and Teaching Center, Ohio, U.S.A.) et le $D^{r}$ G. W. RIEck (Institut für Zuchthygiene und veterinar medizinische Genetik, Justus Liebig - Universität - Giessen, R.F.A.), pour leurs commentaires en ce qui concerne la rédaction du nanuscrit.

\section{Summary}

\section{The C-banding pattern of the Domestic Horse (Equus caballus) the Donkey (Equus asinus) and the mule}

Constitutive heterochromatin (C-band) is evident in all chromosomes of the domestic horse (Equus caballus) except in the eleventh pair. The $\mathrm{X}$ chromosome has a pericentromeric $\mathrm{C}$-band and an interstitial one in the medial part of each of its long arms. The $\mathrm{Y}$ chromosome is almost entirely darkly stained constitutive heterochromatin.

In the Donkey (Equus asinus) constitutive heterochromatin when present is either pericentromeric, telemeric or medial. The $\mathrm{X}$ chromosome has an interstitial C-band in the medial area of each long arm. It is possible to identify nearly all pairs using their morphology, size and C-banding patterns.

In the mule the two haploid sets are easily identified.

\section{Références bibliographiques}

Benikschke K., Brownhil. I. E., BeAth N. N., I962. Somatic chromosomes of the Horse the Donkey and their hybrids the Mule and Hinny. J. Reprod. Fertil., 4, 319-326.

BENIRSCHKE K., 1967. Sterility and fertility of interspecific mammalian hybrids in: Comparative aspects of reproductive failure, 218-234, Int. Conf. Dartmouth Medical School, I966, edited by K. BENIRSCHKE, Springer Verlag, Berlin.

Buckland R. A., Fietcher J. M., Chandiey A. C., i976. Characterization of the domestic horse (Equus caballus) karyotype using G-and C-banding techniques. Experientia, 32, Ix46I 49.

Chandley A. C., Jones R. C., DotT H. M., Alren W. R., ShorT R. V., I974. Meiosis in interspecific equine hybrids. I The male mule (Equus asinus $\times E$. caballus) and hinny (E.caballus $\times$ E. asinus). Cytogenet. Cell. Genet., 13, 330-34I.

Chandley A. C., Short R. V., Alike W. R., I975. Cytogenetic studies of three equine hybrids. J. Reprod. Fert. Suppl., 23, 365-370.

Comings D. E., MATTOCCIA E., I972. DNA of mammalian and avian heterochromatin. Exp. Cell. Res., 71, I I 3-I 3 I.

ELDRIDGE F., BlazAK W. F., 1976. Horse, ass and mule chromosomes. The J. Hered., 67, $361-367$.

Hsu T. C., ARrighi F. E., I97I. Distribution of constitutive heterochromatin in mammalian chromosomes. Chromosoma, 34, 243-253.

Kofman-Aliaro S., Marquez M. H., Mercado R. H., Funes C. F., ig66. Estudio cromosomico en una familia de equidos; yegua caballo y mula. Boln Inst. Estud. Med. Biol. Univ. Nac. Auton. Mex., 24, I47-I57.

Makino S., Sofuni T., SASA KI N. S., 1963. A revised study of the chromosomes in the horse, the ass and the mule. Proc. Japan. Acad., 39, 176-181.

MelchioR I., Hohn H., I976. Der karyotype des Pferdes (Equus caballus) dar gestellt mit Hilfe der G- and C-Bandentechnik. Giessener Beitr. Erbpath. Zuchthyg., 6, I79-I94.

Moorhead P. S., Noweli, P. C., MEliman W. J., Battips D. M., Hungereord D. A., I96o. Chromosome preparations of leucocytes cultured from human peripheral blood. Exp. Cell. Res., 20 6г3-6г6. 
RAman R., Sharma T., I974. DNA replication G and C bands and meiotic behaviour of supernumerary chromosomes of Rattus rattus (Linn.). Chromosoma, 45, III-II9.

Rothfels K. H., Axelrad A. A., Siminovitch L., McCulloch E. A., Parker R. C., i959. The origin of altered cell lines from mouse, monkey and man, as indicated by chromosome and transplantation studies. Canadian cancer Conference (R. W. BIGG, editor, Academic Press, New-York), 3, I89-2I 4 .

Ryder O. A., EPer, N. C., BenirschKE K., r978. Chromosome banding studies of the Equidae. Cytogenet. cell Genet., 20, 323-350.

SaSAKi M. S., Makino S., I962. Revised study of the chromosomes of domestic cattle and the horse. In somatic cells in vitro. J. Hered., 53, 157-I62.

Sinha A. K., Kakati S., Pathak S., I972. Exclusive localization of C-bands in Opossum sex chromosomes. Exp. Cell Res., 75, 265-267.

Sumner A. T., 1972. A simple technique for demonstrating centromeric heterochromatin. Exp. Cell Res., 75, 304-306.

Trujilio J. M., Stennus C., Christian L. C., Onno S., 1962. Chromosomes of the horse, the donkey and the mule. Chromosoma, 13, 243-248. 